\title{
Ship impact study: Analytical approaches and finite element modeling
}

\author{
Pawel Woelke ${ }^{\mathrm{a}, *}$, Najib Abboud ${ }^{\mathrm{a}}$, Darren Tennant ${ }^{\mathrm{b}}$, Eric Hansen $^{\mathrm{b}}$ and Chad Mcarthur ${ }^{\mathrm{b}}$ \\ ${ }^{\mathrm{a}}$ Weidlinger Associates, Inc., New York, NY, USA \\ ${ }^{\mathrm{b}}$ Weidlinger Associates, Inc., 6301 Indian School Rd NE, Albuquerque, NM, USA
}

Received 24 April 2010

Revised 11 May 2011

\begin{abstract}
The current paper presents the results of a ship impact study conducted using various analytical approaches available in the literature with the results obtained from detailed finite element analysis. Considering a typical container vessel impacting a rigid wall with an initial speed of 10 knots, the study investigates the forces imparted on the struck obstacle, the energy dissipated through inelastic deformation, penetration, local deformation patterns, and local failure of the ship elements. The main objective of the paper is to study the accuracy and generality of the predictions of the vessel collision forces, obtained by means of analytical closed-form solutions, in reference to detailed finite element analyses. The results show that significant discrepancies between simplified analytical approaches and detailed finite element analyses can occur, depending on the specific impact scenarios under consideration.
\end{abstract}

Keywords: Ship impact, collision, ship crashworthiness

\section{Introduction}

Ship impact is an important problem in the analysis and design of bridges, oil platforms, and other marine structures. Designing the structure to resist ship collision requires knowledge of the loads imposed on the structure by the vessel. Accurate determination of these loads is, therefore, very important for optimal design. Design practice typically requires simple and effective methods of loads determination. Unfortunately, it is difficult to accurately calculate the impact forces imparted on the bridge or other marine structure without detailed analysis of the impact itself. In this paper, potential discrepancies between the impact loads calculated using available analytical methods and detailed finite element analysis are investigated. A significant number of parameters influence ship crushing loads. The response of the structure subjected to a vessel impact is largely dependent on the size and mass of the vessel, the local stiffening pattern (a vessel with longitudinal stiffeners exerts higher forces on the struck obstacle than a vessel with transverse stiffeners), location and function of the bulkheads, possible ice-strengthening classification, draught, presence of the bulbous bow, and many other factors. Most analytical methods neglect the majority of these factors. Moreover, despite universal application of design methods to a variety of vessels, many methods were validated using data obtained from collisions of specific classes of ships. These and other factors can greatly affect the accuracy of the calculated responses, as shown below.

The paper consists of 5 Sections. In Section 2, a brief overview of the analytical methods for determination of the loads and energies involved in ship impact is presented. In Section 3, the parameters influencing impact forces are discussed. In Section 4, a finite element model of the typical container ship is presented, with comparison of the results between the analytical and numerical analyses. The summary and conclusions are given in Section 5.

\footnotetext{
${ }^{*}$ Corresponding author: Pawel Woelke, Weidlinger Associates, Inc., 40 Wall Street, New York, NY 10005-1304, USA. Tel.: +1 212367 2983; Fax: +1 212497 2483; E-mail: woelke@wai.com.
} 


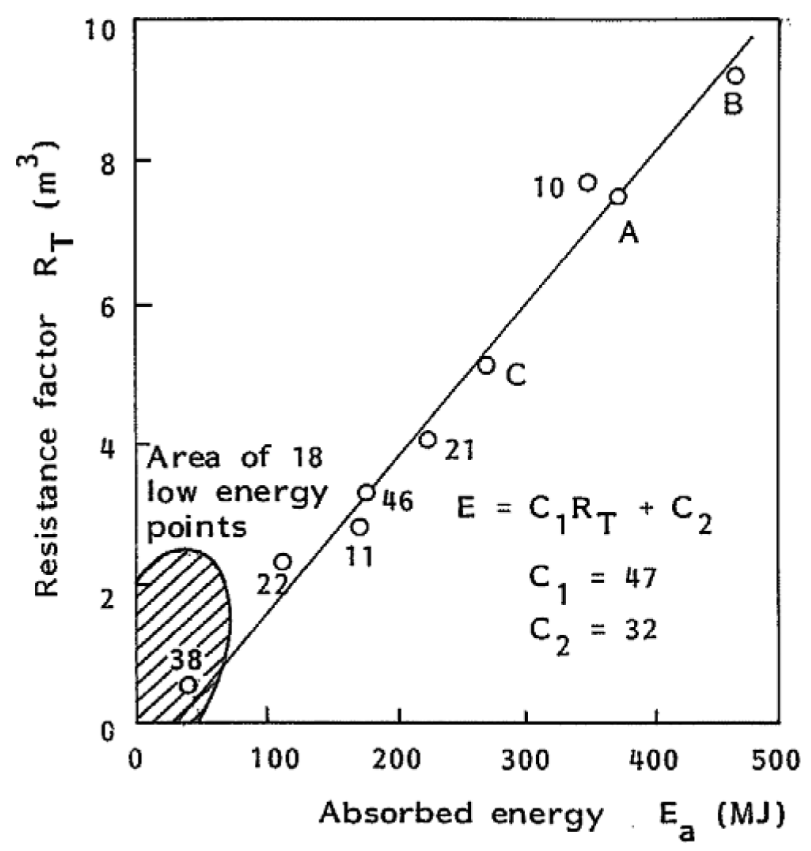

Fig. 1. Correlation between energy absorbed in collision and volume of damaged material - Minorsky [7].

\section{Overview of the design methods and analytical approaches}

\subsection{Minorsky's method}

One of the first practical methods for calculating ship-crushing loads was proposed by Minorsky in 1959 [7]. Based on the investigation of a number of ship-to-ship collisions, a linear relationship was established between the volume of deformed steel and the energy dissipated in the deformation process. The relation allows for determination of the ship penetration into the struck obstacle before all the initial kinetic energy has been absorbed. Correlation between the absorbed energy and volume of deformed steel is shown in Fig. 1.

The resistance factor $\mathrm{R}_{T}$ in Fig. 1 (vertical axis) is calculated as the volume of the ship components with depth in the direction of penetration (e.g. decks, flats in both ships, longitudinal bulkheads and components of the striking vessel [3]. As shown in Fig. 1, the area of low-impact energy is associated with significant scatter of the data points. This is the region where local effects become important. For larger energies, the relationship proposed by Minorsky closely represents the collected data points.

Minorsky's method was based on the collisions of similar ships, which did not necessarily represent the entire spectrum of vessels likely to be involved in collision. Nevertheless, the volume of steel deformed during impact is part of the equation that provides information about the bow structure. The volume of deformed steel is ship-specific and accounts, although indirectly, for differences in the bow construction. This method is therefore ship-specific, which distinguishes it from some of the other methods.

Only the relation between the energy and volume of deformed steel, as well as the average force values, can be determined using Minorsky's method. The accurate force-time or force-penetration functions cannot be directly derived. The effect of the variation of impact angles and limited width of the impacted structure are not considered. These factors can significantly influence the response of both the impacting vessel and the impacted structure.

\subsection{Amdahl's method}

Amdahl's method was based on determination of the energy dissipated in the process of deformation of the key structural components of the bow, such as angles, T-sections, and cruciforms [3] (see also [12]). The cumulative 


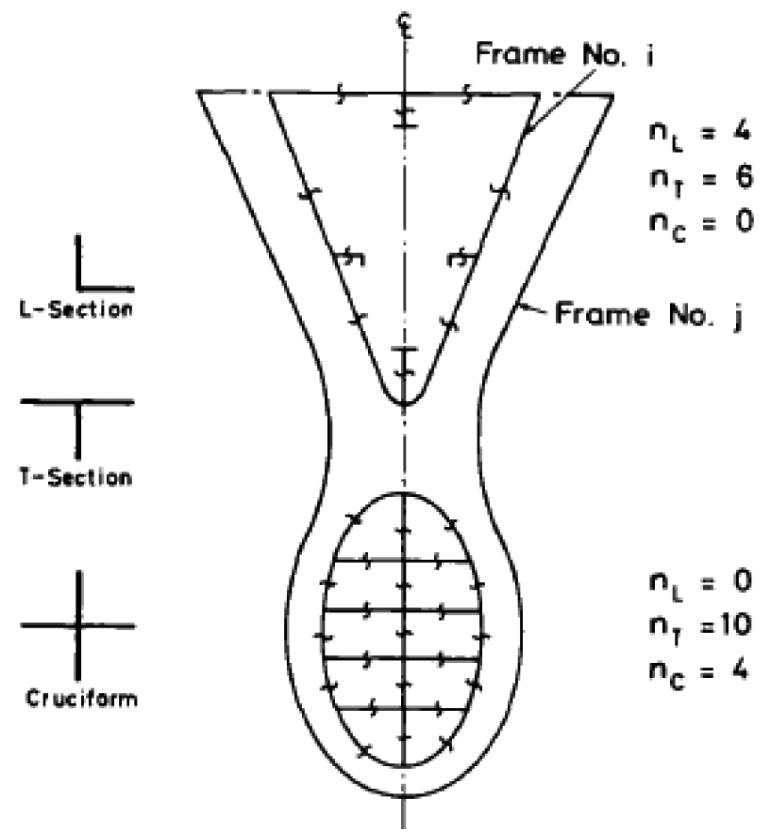

Fig. 2. Cross-section of the bow with basic structural sections [8].
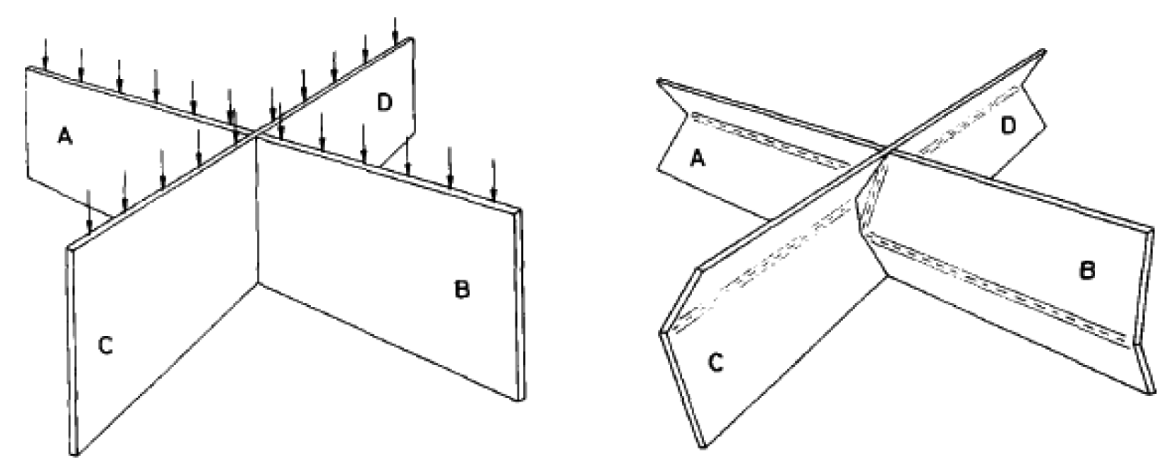

Fig. 3. Crushing mechanism for a cruciform structural element [8].

energy contributions from all those basic structural elements of a specific cross-section of the bow give the total crushing force imparted on the structure by the vessel. The length of each basic structural element and its crushing load are determined by minimization of the energy dissipated through inelastic deformation [3]. The typical cross section of the bow with basic structural elements is shown in Fig. 2 [8]. The basic crushing mechanism of the cruciform section is shown in Fig. 3.

Based on the subsequent cross-sections considered and the increasing number of structural elements, the relation between load and penetration is determined. Amdahl's expression is given by Eq. (1) [3]:

$$
\sigma_{c}=2.42 \sigma_{o}\left[\frac{n_{A T} t^{2}}{A}\right]^{0.67}\left[0.87+1.27 \frac{n_{c}+0.31 n_{T}}{n_{A T}}\left(\frac{A}{\left(n_{c}+0.31 n_{T}\right) t^{2}}\right)^{0.25}\right]^{0.67}
$$

where: 


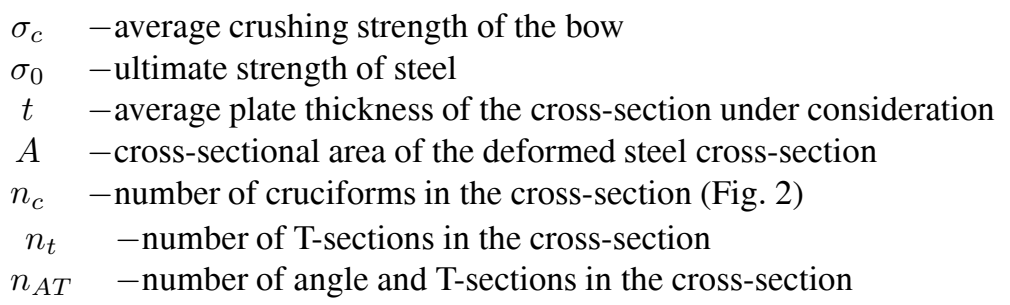

The total crushing load is found by multiplying the above stress by the cross-sectional area of the deformed steel material:

$$
P_{c}=\sigma_{c} A
$$

The load-penetration relation can be determined using the above equations, as long as the details of the bow construction are known. In that respect, Amdahl's method is similar to Minorsky's, i.e., both methods are shipspecific. With Amdahl's method, however, much more detailed information about the stiffening pattern is necessary for accurate results. The local effects become less important for large impact energies.

Eccentric impact scenarios and limited width of the impacted structure are not considered.

\subsection{Pedersen's method}

Pedersen et al. [8] proposed a set of empirical expressions for calculation of the bow collision forces, maximum penetration, and impact duration. The collision forces are determined as functions of the vessel size and initial speed, as well as bow profile. Pedersen's empirical expression for bow collision forces is given by [8]:

$$
P_{\text {bow }}=\left\{\begin{array}{lrr}
P_{0} \bar{L}\left[\bar{E}_{i m p}+(5.0-\bar{L}) \bar{L}^{1.6}\right]^{0.5} & \text { for } \quad \bar{E}_{i m p} \geqslant \bar{L}^{2.6} \\
2.24 P_{0}\left[\bar{E}_{i m p} \bar{L}\right]^{0.5} & \text { for } \quad \bar{E}_{i m p}<\bar{L}^{2.6}
\end{array}\right.
$$

where:

$$
\begin{aligned}
& \bar{L}=L_{p p} / 275 m \text { (normalized, empirical length) } \\
& \bar{E}_{i m p}=E_{i m p} / 1425 \mathrm{MNm} \text { (normalized, empirical energy) } \\
& E_{i m p}=\frac{m_{x} V_{0}^{2}}{2} \\
& P_{b o w} \quad \text {-maximum bow collision load }[\mathrm{MN}] \\
& P_{0} \quad \text {-reference collision load } P_{0}=210 \mathrm{MN} \\
& E_{i m p} \quad \text {-energy absorbed by inelastic deformation } \\
& L_{p p} \quad \text {-length of the vessel } \\
& m_{x} \quad \text { - mass plus }(5 \%) \text { added mass with respect to longitudinal motion }\left[10^{6} \mathrm{~kg}\right] \\
& V_{0} \quad \text {-initial speed of the vessel [m/s] }
\end{aligned}
$$

From the energy balance, the maximum penetration can be determined (see reference [8] for details of the derivation):

$$
s_{\max }=\frac{\pi}{2} \frac{E_{i m p}}{P_{\text {bow }}}
$$

The impact duration is given by:

$$
T_{0} \approx 1.67 \frac{s_{\max }}{V_{0}}
$$

One disadvantage of Pedersen's method is that the calculation of the penetration is based on the maximum collision force. This is equivalent to the assumption that the impact force remains constant after reaching its maximum. The forces, however, can vary significantly with time during the impact. Examples of crushing force-time histories calculated using detailed finite element analyses are shown in Fig. 4. The finite element analyses performed to determine the force-time histories are described below.

Another important characteristic of Pedersen's method is that it does not include details of the bow construction. This can compromise the accuracy or limit the applicability of the method to the classes of ships used to collect the 


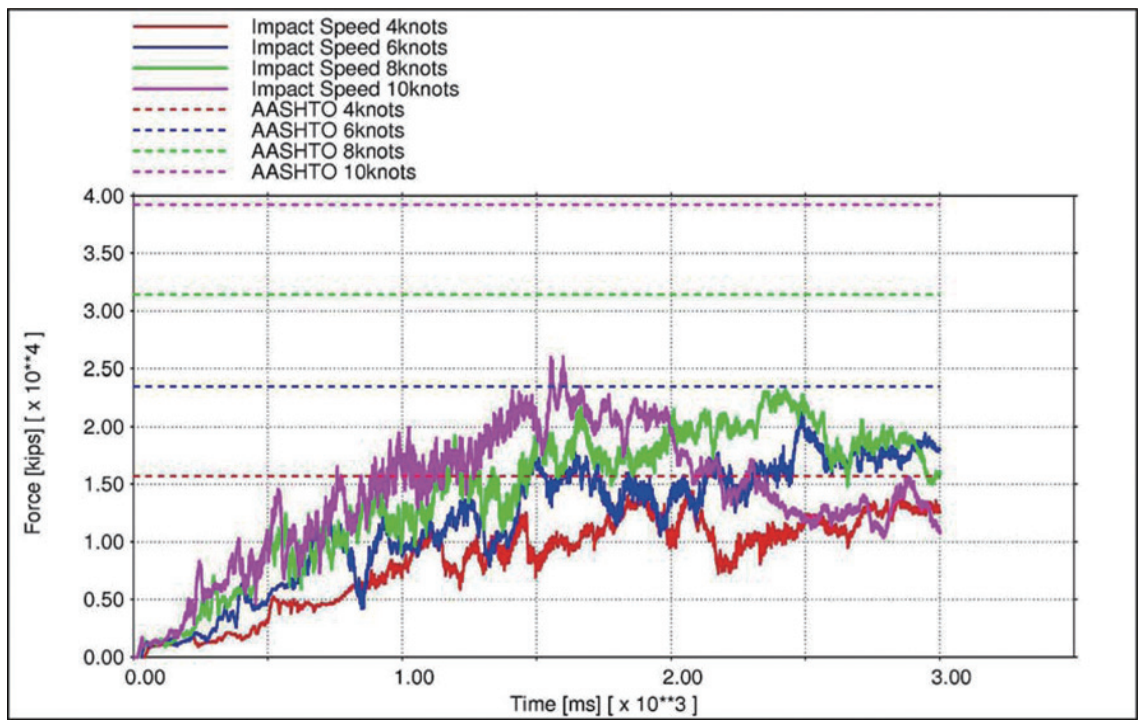

Fig. 4. Time history plots of the vessel crushing force for various impact forces - calculated using a detailed finite element ship model.

data to develop Eqs (3)-(5). As Pedersen's estimates were largely based on vessels meeting various Baltic Ice Class requirements, they may lead to very conservative results when used to analyze impacts involving vessels with more flexible bow construction.

\subsection{Other analytical approaches}

Numerous other methods of determining ship collision forces are available in the literature. The most popular ones are listed and briefly described below. Detailed descriptions of some of the methods appear in reference [8].

- Gerard [4] - Semi-empirical method for determination of crushing forces established by correlation between the results of a series of simple panel tests with various stiffeners. This method, which is similar to Amdahl's, and it has been found suitable for many industrial applications.

- Yang and Caldwell [14] - A method similar to Amdahl's, based on estimation of the energy dissipated in the process of inelastic deformation. Both methods use the folding mechanisms proposed by Wierzbicki [12].

- Saul and Svensson [11] - A universal empirical method used to estimate the crushing force as a function only of the weight of the vessel:

$P_{\text {bow }}=0.88 \sqrt{D W T} \pm 50 \%$

$P_{\text {bow }} \quad$-maximum bow collision load [MN]

$D W T$-deadweight of the vessel in metric tones

The factor $\pm 50 \%$ indicates large uncertainties associated with the force predictions. Possible variations of the bow construction, angle of incidence, limited width of the obstacle, or other factors are not accounted for.

- AASHTO Specification [1] - Similar approach to Saul and Svensson. Only mass and initial velocity of the ship influence the calculated collision force. AASHTO formulas are given by:

$$
\begin{aligned}
P_{\text {bow }} & =0.12 V_{0} \sqrt{D W T} \\
s_{\max } & =\frac{\pi}{2} \frac{E_{\text {imp }}}{P_{\text {bow }}}
\end{aligned}
$$




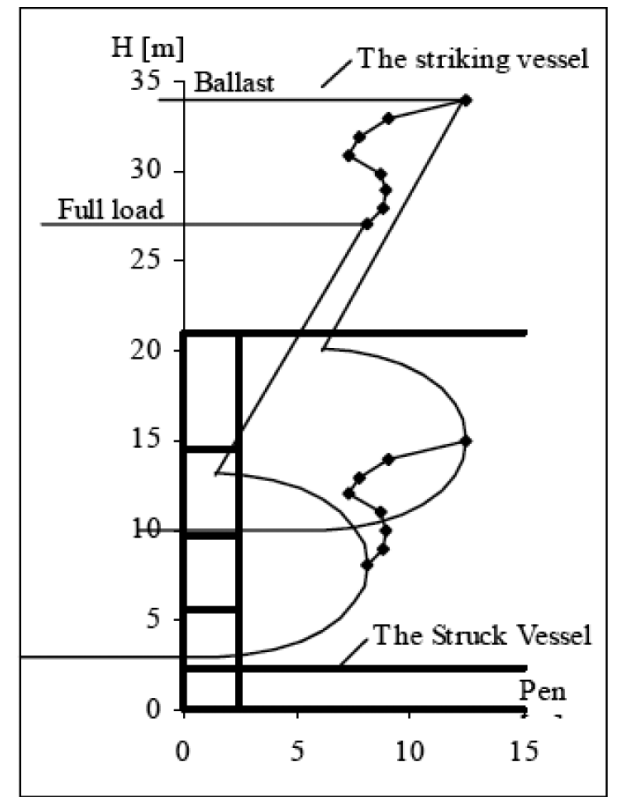

Fig. 5. Penetration of the striking vessel into a struck vessel [6].

$P_{\text {bow }} \quad-$ maximum bow collision load [MN]

$V_{0} \quad$-initial ship velocity $[\mathrm{m} / \mathrm{s}]$

$D W T$-deadweight of the vessel in metric tons

$s_{\max } \quad$-maximum penetration - (4)

$E_{i m p} \quad$-energy absorbed by inelastic deformation

Possible variations of the ship construction or boundary conditions have not been accounted for.

\section{Factors influencing the impact forces}

A variety of factors influence the response of both the impacted structure and the vessel to collision. Identification and consideration of these factors is very important, even if they ultimately lead to the neglect of certain phenomena as not critical for specific problems. Some important factors to be considered in ship impact analyses are:

- The local stiffening pattern and other details of the bow construction often play a significant role. Stiffer bows naturally produce higher crushing forces, imposing higher loads on the impacted structure.

- Large tankers usually have a double hull with extensive stiffening.

- Ice-strengthened bows of large ships (DWT>40,000 tons) are generally considered rigid [6]. This typically shifts the inelastic deformation away from the stiffest regions of the bow.

- The ballast condition (as opposed to fully loaded) is most often a critical impact condition for large and/or ice-stiffened ships (Fig. 5). Ships in ballast have their forepeak tanks filled with water, which increases the impact forces significantly.

- Although the bulbous bow shape is prevalent in most of the world's fleet [5], some tankers and bulk carriers do not have bulbous bows. This can be decisive in the case of impacting bridges or oil rigs, as it often determines which part of the structure is impacted first.

- Geometric variations of the impacted structures is also very important. The impacted obstacles are often not flat and are of finite width (bridges, oil rigs). Most of the analytical methods used to determine impact forces consider the rigid wall impact scenario. Impacting a flexible structure usually results in smaller impact forces and longer impact duration [5]. 


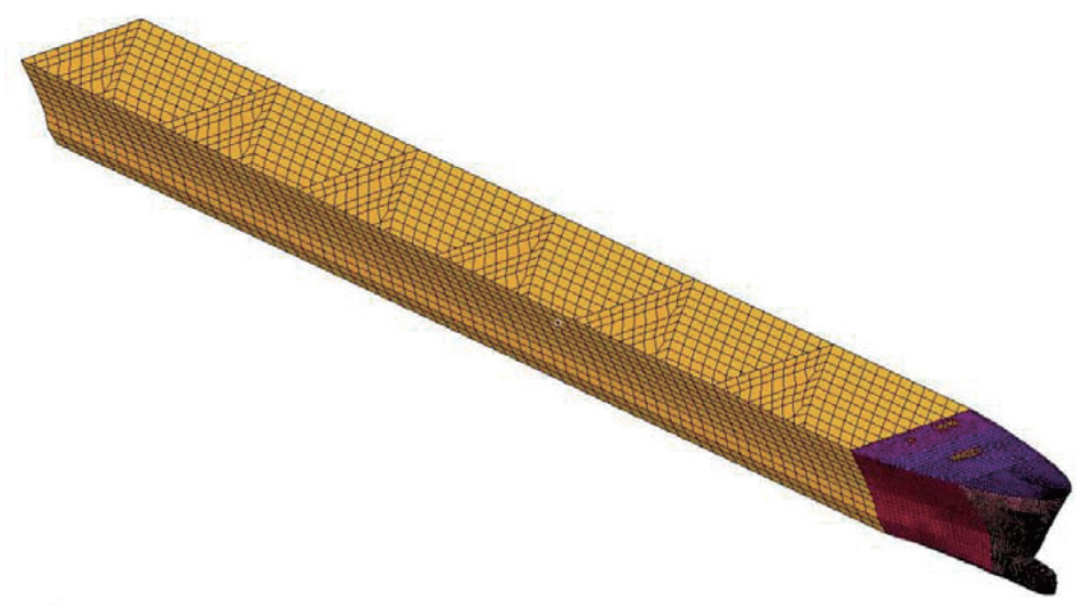

Fig. 6. Finite element model of the container vessel used in the analyses.

- Variation of angle of incidents is rarely considered in the analytical approaches. The head-on collision is usually considered as a worst-case scenario. Navigational conditions, however, often make this scenario impossible. In such cases, designing the impacted structure to resist forces resulting from a head-on collision is overly conservative.

Many other factors also should be investigated in a ship impact study. Accounting explicitly for all of them, however, is neither realistic nor economical, especially for the designer. Simplified design methods are most often a compromise between the required accuracy on one hand and universality and efficiency of the method on the other hand. The choice to neglect certain phenomena should be a conscious one, as the differences between particular approaches, including the detailed finite element analysis, can result in large differences in calculated response, as shown in Section 4.

\section{Finite element analysis}

A ship collision is considered, where a typical container vessel impacts a rigid wall. The penetration, crushing load, and energies are calculated using finite element analysis, and the results are compared with those obtained using the analytical methods described above.

The analysis is performed by means of a nonlinear dynamic transient analysis finite element code NLFlex, developed and maintained by Weidlinger Associates. The program uses a technologically advanced first-principles approach to modeling a structure's response to extreme loading conditions. NLFlex has been extensively validated through pretest predictions and post-test correlation with small- and large-scale tests for a large number of impact, airblast, ground shock, and thermal loading situations.

\subsection{Finite element model}

An 80000 tons DWT, 247m long typical container vessel is considered. The finite element model of the ship is built by means of approximately 25758 shell elements. The finite element model of the vessel is shown in Fig. 6.

The model of the vessel is divided into two sections. The front section of the ship extends from the bow, through the collision bulkhead to the hold bulkhead, for a length of approximately 135 feet. The rear section extends from the collision bulkhead to the stern and is approximately 675 feet. Only the front section of the ship is directly involved in impact energy dissipation; the rear of the ship provides the mass with inertia. The front section is modeled in much greater detail, by means of 23000 shell elements (compared to 2500 elements in the rear section). The bulkheads, decks, and most important stiffeners are modeled explicitly. Details of the finite element model of the front section of the vessel are shown in Fig. 7. 


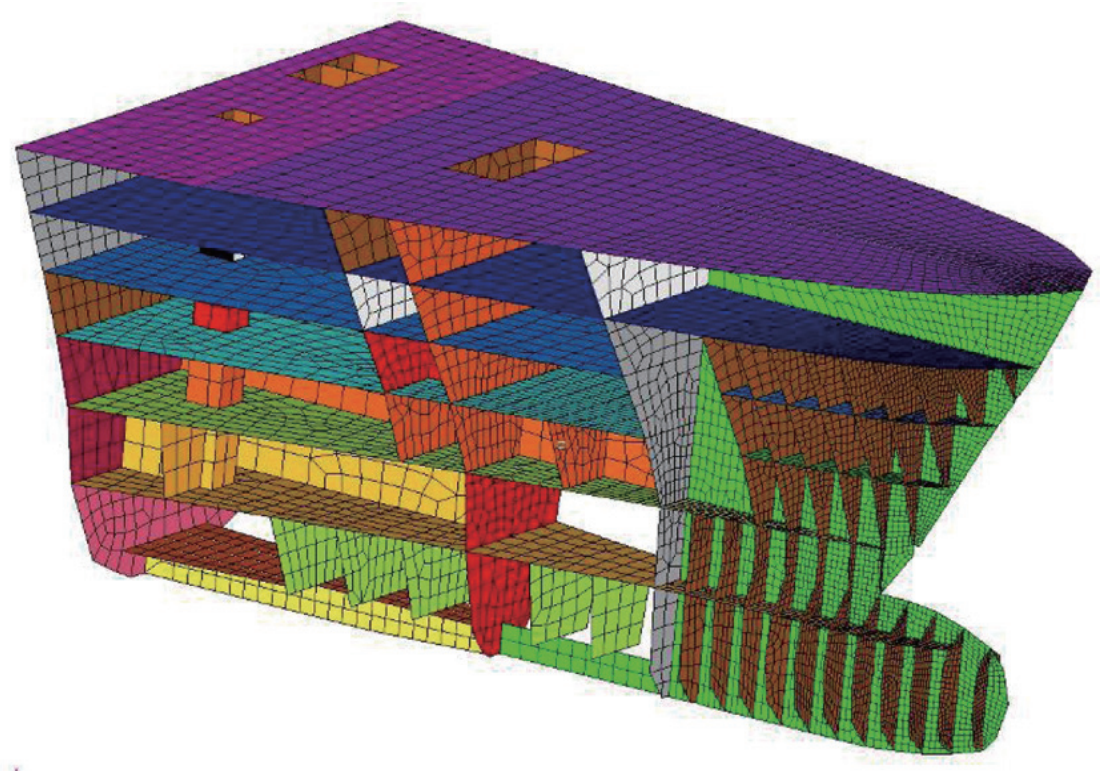

Fig. 7. Finite element model of bow of the container vessel used in the analyses.

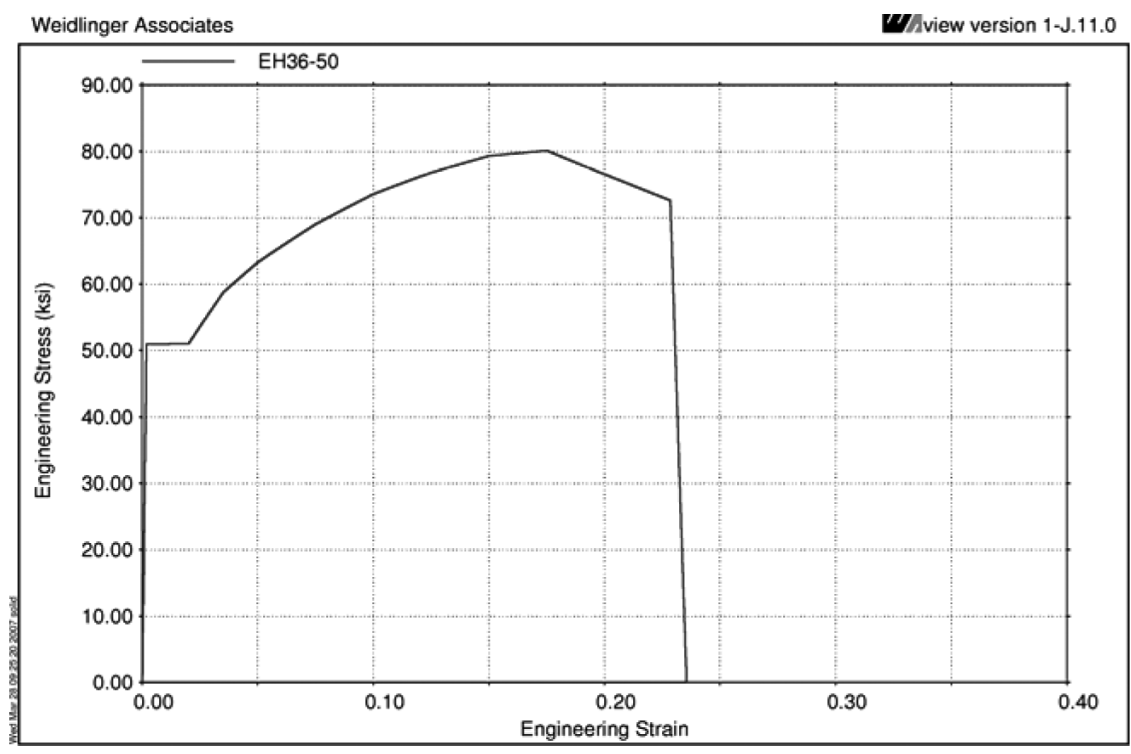

Single Solid Element Test

Fig. 8. Engineering stress-strain curve for EH36-50 steel used in the finite element analysis.

EH36 steel was adopted in the finite element model, which is a typical steel used in the construction of container ships. The parameters characterizing EH36 steel appear in reference [2]. The engineering stress-strain curve of EH36 steel is shown in Fig. 8.

\subsection{Analysis results}

A container vessel with an initial velocity of $10 \mathrm{knots,} \mathrm{impacting} \mathrm{a} \mathrm{rigid} \mathrm{wall} \mathrm{is} \mathrm{investigated.} \mathrm{The} \mathrm{velocity} \mathrm{of}$ the ship is not maintained, i.e., the engines are assumed to be shut down just before the impact. The results of this 

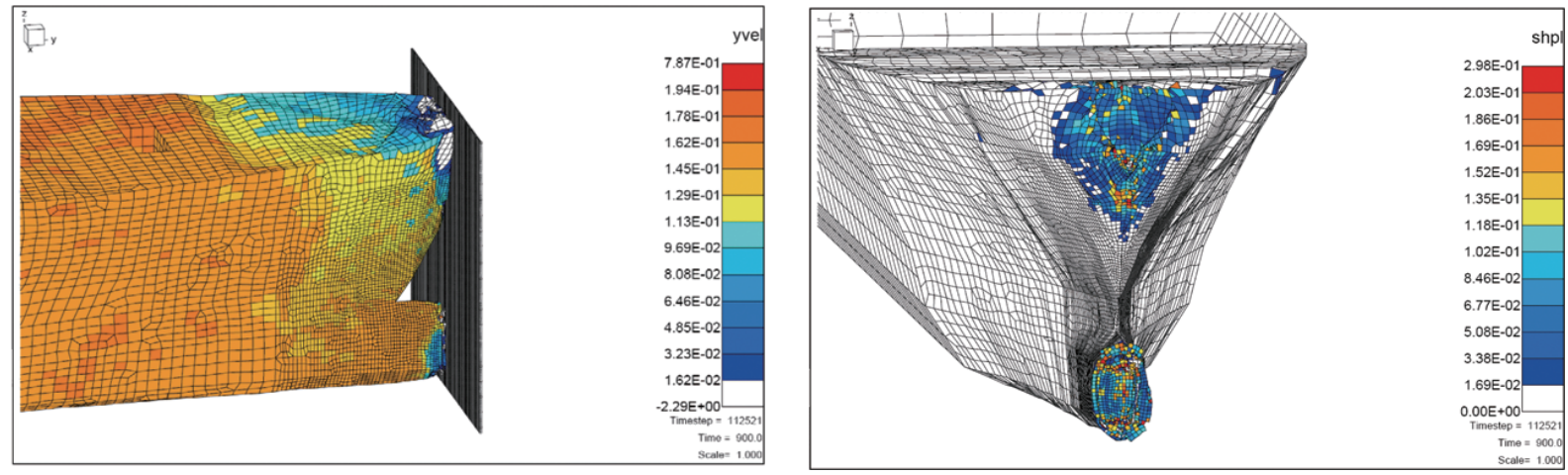

Fig. 9. Ship velocity at $0.9 \mathrm{~s}$ (impact at $0.0 \mathrm{~s}$ ) and equivalent plastic strains in the bow.
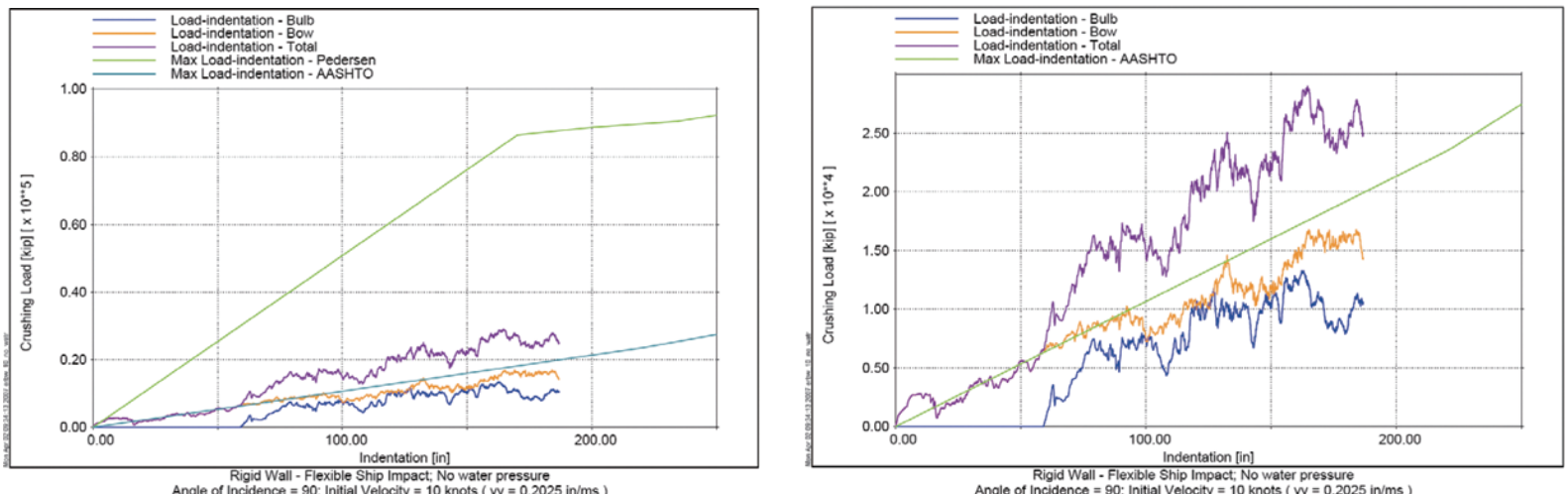

Fig. 10. Impact Load-Indentation history plots - comparison between numerical and analytical results (bottom plot - reduced crushing load scale).

analysis are compared with those obtained using the analytical methods. The contour plot of ship velocity at $0.9 \mathrm{~s}$ and equivalent plastic strains are shown in Fig. 9.

At $0.9 \mathrm{~s}$, after impact only the forepeak section of the bow, which is directly involved in impact, has either come to a stop upon contact with the rigid obstacle or has slowed down significantly. Most of the volume and mass of the ship is still moving with a velocity close to the initial velocity of 10 knots. The inelastic deformations are fairly extensive, and the volume of deforming steel is increasing. This indicates that the impact duration is most likely in excess of 5 seconds and that accurate representation of the collision would require that the analysis include this time. The comparison between the collision forces calculated using analytical methods and those calculated using detailed finite element provides useful information, even in the early stages of the analysis.

Figure 10 compares Load-Indentation functions obtained using numerical and analytical methods. A close correlation between the forces predicted by the AASHTO guide and those calculated using numerical methods is observed. In the same time, Pedersen's method predicts much higher forces.

Figure 11 shows the relations between the vessel energy lost and indentation. Again, a close relation is observed between the numerical predictions and the AASHTO guide, as well as Minorsky's method, while Pedersen's method predicts significantly higher energy. Both AASHTO and Pedersen's method are of the "one size fits all" type, where the forces calculated are the function only of the initial speed and size of the vessel. Variations in stiffening pattern are not accounted for. The major difference between AASHTO and Pedersen's method is that, as the latter was developed for vessels with ice-strengthening classification, it predicts much higher collision forces.

Minorsky's method is vessel-specific, i.e., it accounts for variations in stiffening pattern. Calculating the vessel response using Minorsky's method requires knowledge of the stiffener's size and spacing, to access the volume of steel deforming during the collision. The results obtained with Minorsky's method compare very well with the numerical results. 

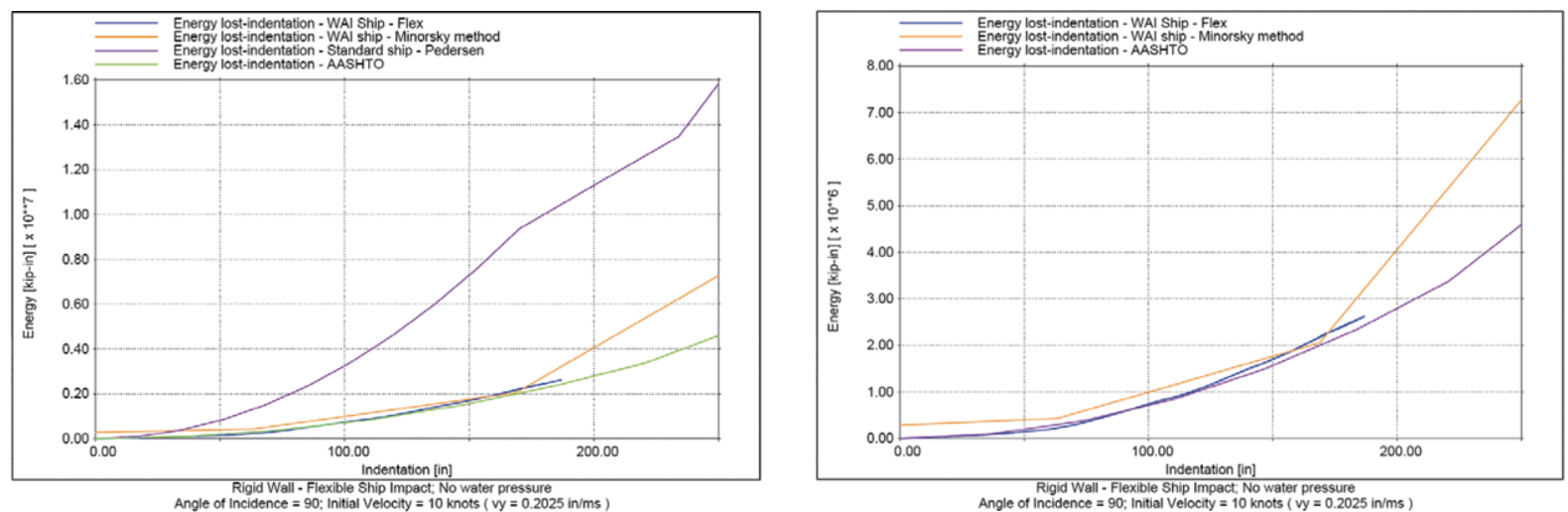

Fig. 11. Energy lost-Indentation history plots - comparison between numerical and analytical results (bottom plot - reduced energy scale).

Although there is close correlation among the AASHTO, Minorsky's, and numerical results, there is also a large discrepancy between Pedersen's and all the other results. This is because Pedersen's method was intended to cover various classes of ships, including those with ice-strengthened bows. It will most likely produce an upper bound solution of the collision forces, which in some instances is a few hundred \% higher than the actual forces. AASHTO also provides a simple approach applied universally to a variety of ships. For a given ship size, initial velocity, and stiffening pattern, both AASHTO and finite element analyses can produce similar responses, as is the case here. If, however, a different class of ship were analyzed, with only a slight variation of weight the discrepancy between the numerical and AASHTO results could be far greater.

Moreover, all the analyzed impact scenarios are practically the same in terms of the geometry of the impacted structure. In all the cases presented here, the ship was assumed to impact a rigid wall. In reality, the collision often occurs between the vessel and a marine structure that is not flat and is of limited width. These variations in boundary conditions would make no difference in the results produced by analytical design methods. The only reliable way to consider such variations is through detailed analysis of the specific problem.

\section{Summary and conclusions}

The results of both numerical and analytical predictions of ship collision forces indicate that simplified design methods can lead to overly conservative designs. More important, in certain instances unconservative designs may result from such considerations. There is a need in the design community for a quick, easy, and universal method to reliably predict the loads exerted on both impacted and impacting structure during a collision. In light of the complexity of the problem, however, obtaining accurate results by means of a simple and universal design method is very difficult, if even possible.

Ship collisions should be investigated in a detailed fashion, on a case-by-case basis. Designing a structure to resist ship impact, designing a protective structure, and investigating a collision event that has occurred all require solution of a specific problem, accounting for as many factors influencing the structural response as possible, to deliver an accurate and cost-effective solution. The simplified analytical design methods should be used with caution and, in most cases, treated as the 'back-of-the-envelope' preliminary solution.

\section{References}

[1] AASHTO, Guide Specification and Commentary for Vessel Collision Design of Highway Bridge, GVCB-1. American Association of State Highway and Transportation Officials, Washington, DC, 1991.

[2] ASTM A131/A131M-08, Standard Specification for Structural Steel for Ships, 2008.

[3] J. Amdahl, Energy Absorption in Ship-Platform Impacts. Dr. Ing. Thesis, Report No. UR-83-84, The Norwegian Institute of Technology, Trondheim, 1983. 
[4] G. Gerard, Compressive Strength of Flat Stiffened Panels. National Advisory Committee for Aeronautics, NACA TN3785, 1957.

[5] O.D. Larsen, Ship Collision with Bridges. The Interaction between Vessel Traffic and Bridge Structures. Structural Engineering Documents 4. IABSE, Zurich, 1993.

[6] M. Lutzen, B.C. Simonsen and P.T. Pedersen, Rapid Prediction of Damage to Struck and Striking Vessels in a Collision Event. SSC/SNAME/ASNE Ship Structure Symposium, June 2000.

[7] V.U. Minorsky, An analysis of ship collisions with reference to nuclear power plants, J Ship Res 3(2) (1959), 1-4.

[8] P.T. Pedersen, S. Valsgard, D. Olsen and S. Spangenberg, Ship Impacts: Bow Collisions, Int J Impact Engng 13(2) (1993), $163-187$.

[9] M.J. Petersen, Dynamics of ship collisions, Ocean Engng 9(4) (1982), 295-329.

[10] M.J. Petersen and P. Terndrup Pedersen, Collision between ships and offshore platforms. 13th Annual Offshore Technology Conference, Paper No. OTC 4134, 1981.

[11] R. Saul and H. Svensson, Meansof reducing consequences of ship collisions with bridges and offshore structures. IABSE Colloquium on Ship Collision with Bridges and Offshore Structures, Copenhagen, 1983.

[12] T. Wierzbicki, Crushing behaviour of plate intersections. Structural Crashworthiness (edited by N. Jones and T. Wierzbicki); Chapter 3; Butterworth and Co., London, 1983.

[13] G. Woisin, Design against collision. Proc. Int. Symposium on Advances in Marine Technology. Vol. 2, Trondheim, 1979.

[14] P.D.C. Yang and J.B. Caldwell, Collision energy absorption of ships' bow structures, Int J Impact Engng 7(2) (1988). 

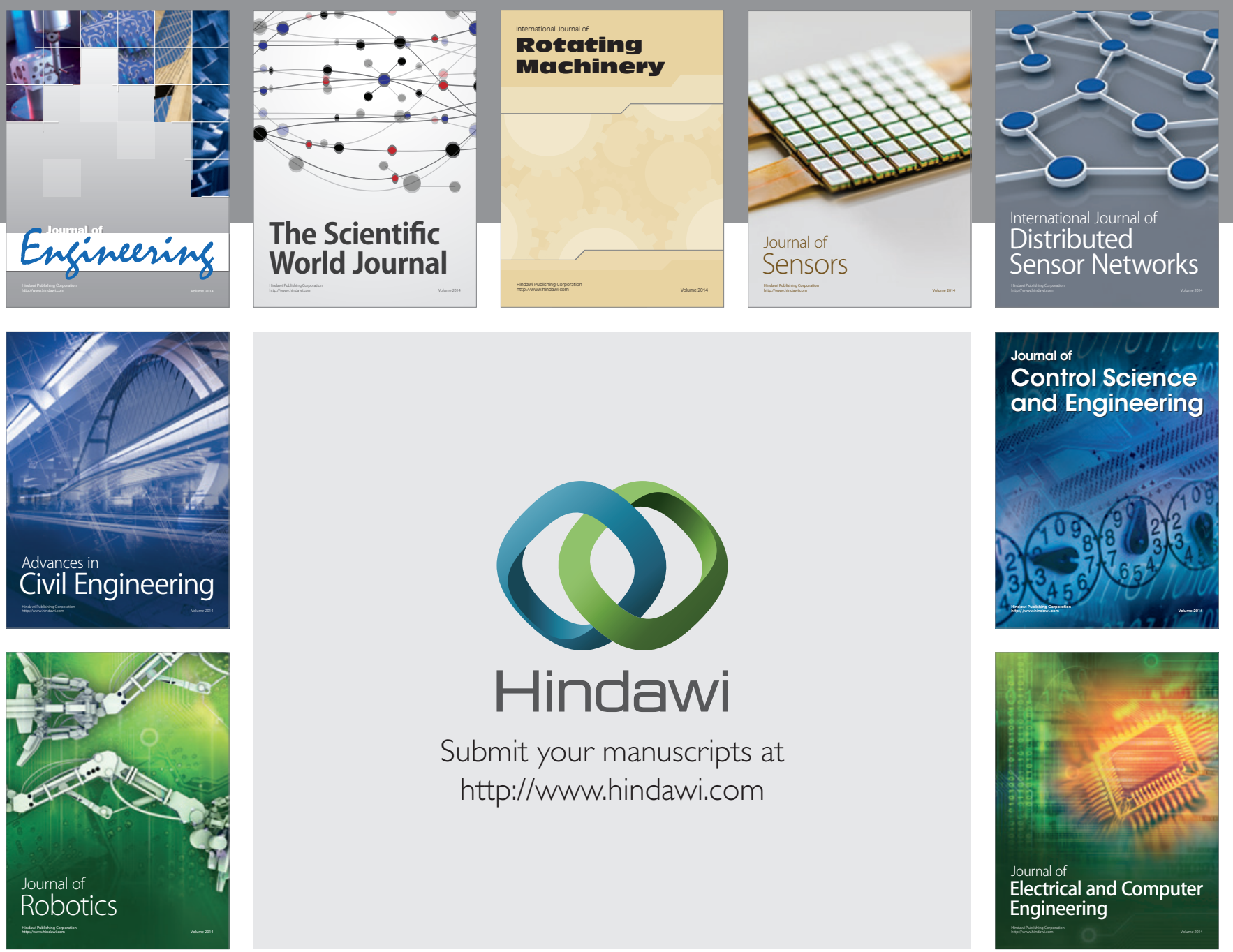

Submit your manuscripts at

http://www.hindawi.com
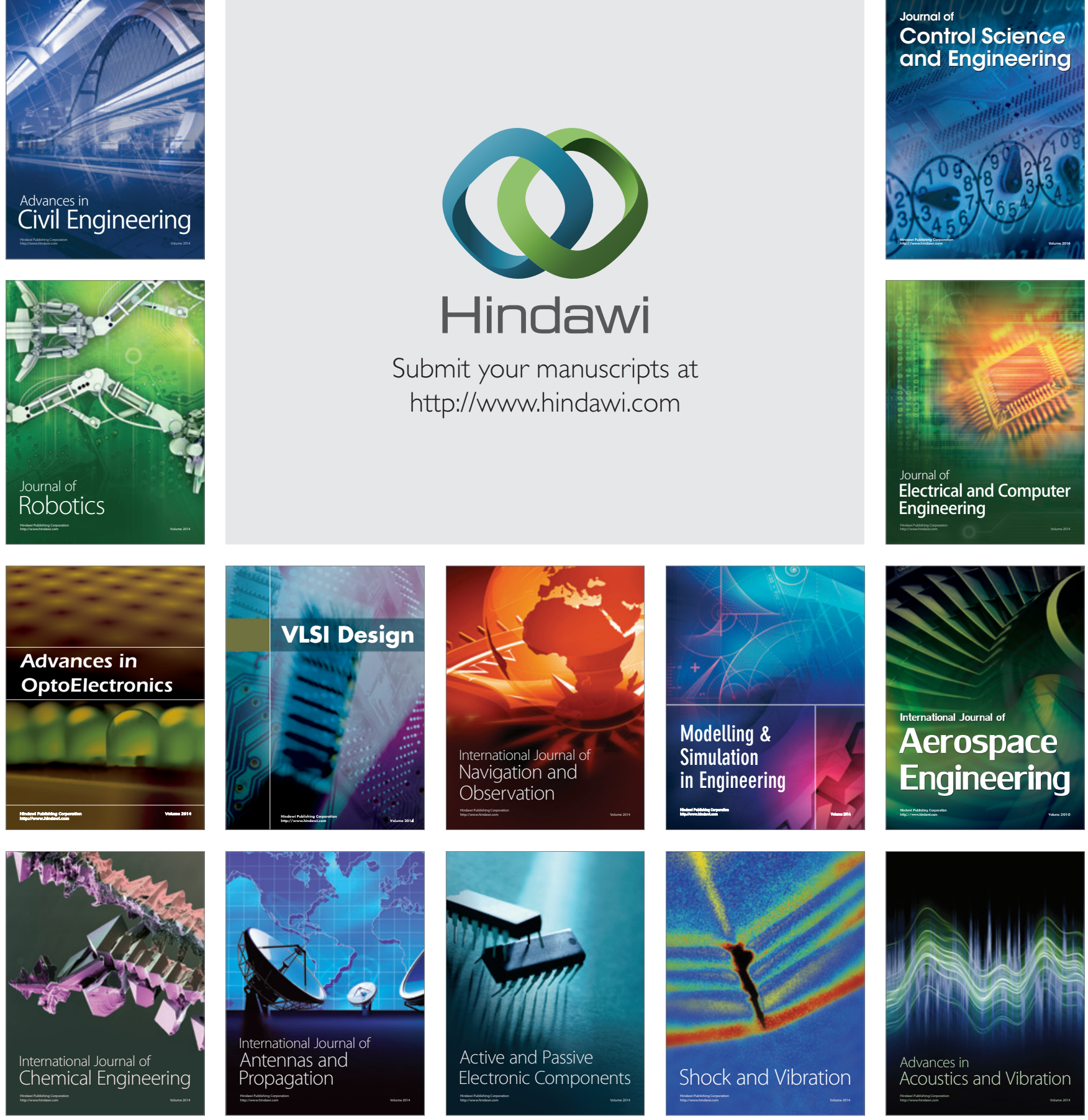